\title{
AN ANALYSIS AND COMPARISON OF BRAINSTEM AUDITORY EVOKED POTENTIALS AMONG SOUTH INDIAN MIDDLE-AGED AND ELDERLY SUBJECTS AND PATIENTS WITH TYPE II DIABETES MELLITUS
}

\author{
Ch. Venkatasubbaiah ${ }^{1}$, R. Ananth' ${ }^{2}$ S. Muneeruddin Ahmed ${ }^{3}$
}

${ }_{1}$ Associate Professor, Department of ENT, Rajiv Gandhi Institute of Medical Sciences, Kadapa, Andhra Pradesh.

2MASLP, Audiologist and Speech Pathologist, Department of ENT, RIMS, Kadapa, Andhra Pradesh.

3Professor, Department of ENT, Kurnool Medical College, Kurnool, Andhra Pradesh.

ABSTRACT

\section{BACKGROUND}

Despite the evidence that the incidence of hearing impairment is higher in type 2 diabetes subjects, very little is known about the nature and characteristics of this disability and the specific mechanisms leading to the hearing problems in diabetic adults.

\section{MATERIALS AND METHODS}

40 patients with Type 2 Diabetes Mellitus were included in the study and grouped as patients aged 35 to 58 years (A) and aged above 58 years (B); 40 patients were taken as control group with similar age grouping; 35 to 58 years (C) above 58 years (D). Pure Tone Audiometry (PTA) test was conducted according to the ASHA guidelines. Objective hearing evaluation was done by BERA (Brainstem Evoked Response Audiometry).

\section{RESULTS}

Higher degree of Hearing Loss (HL) was observed in DM patients in all frequencies from $500 \mathrm{HZ}$ to $10 \mathrm{KHZ}$ in comparison to nonDM patients and it was statistically significant. Slower rate peaks occurred at I, III and V waves in elderly DM patients when compared to the elderly control group. (Peak I and V $(\mathrm{p}<0.001)$ ). Similarly, in the middle-aged group peaks I, III and V occurred at a significantly slower rate in the DM group when compared to the non-DM group $(\mathrm{p}<0.001)$. Interpeak latencies at 21.1 rate for peak III-V and peak $\mathrm{I}-\mathrm{V}$ were consistently longer among the diabetics groups of two age groups when compared to the control group $(\mathrm{p}<0.001)$. Similar results were obtained at 63.3 rates in the elderly and middle-aged group.

\section{CONCLUSION}

In the earlier course of Type 2 Diabetes, though the person may be asymptomatic degenerative changes may begin to appear in the central auditory pathway and result in significant HL, which could be detected with early investigation to elicit of Brain Stem evoked potentials.

\section{KEYWORDS}

Type 2 Diabetes, Hearing Loss, Auditory Evoked Potentials, Interpeak Latency.

HOW TO CITE THIS ARTICLE: Ch. Venkatasubbaiah, Ananth R, Ahmed SM. An analysis and comparison of brainstem auditory evoked potentials among South Indian middle-aged and elderly subjects and patients with type II diabetes mellitus. J. Evolution Med. Dent. Sci. 2016;5(62):4332-4336, DOI: 10.14260/jemds/2016/989

\section{INTRODUCTION}

Insufficient secretion of insulin combined with the defective uptake of secreted insulin is the hallmark of type 2 diabetes mellitus. ${ }^{1}$ The most common risk factor of type II diabetes is sedentary behaviour, consumption of high calorie food, genetic predisposition and obesity. ${ }^{2}$ Recent evidence suggests that there are over 62 million people with type II diabetes in India and this number is set to escalate rapidly. ${ }^{3}$ Although, diabetes leads to considerable morbidity, it is the long term complications of diabetes like retinopathy, nephropathy, neuropathy, cardiovascular disease, peripheral vascular disease and cerebrovascular disease that cause mortality in diabetes patients. ${ }^{4}$ In the recent decades, hearing impairment has been increasingly recognized as one of the complications of diabetes, particularly in aged people. Over two-thirds of diabetic adults have been known to be affected by hearing

Financial or Other, Competing Interest: None.

Submission 21-06-2016, Peer Review 21-07-2016,

Acceptance 27-07-2016, Published 02-08-2016.

Corresponding Author:

Dr. Ch. Venkatasubbaiah,

Associate Professor,

Department of ENT,

Rajiv Gandhi Institute of Medical Sciences,

Kadapa, Andhra Pradesh

E-mail: venkatasubbaiahent@gmail.com

DOI: $10.14260 /$ jemds $/ 2016 / 989$ impairment and compared to the non-diabetic population and the degree of hearing impairment is about two-fold higher in diabetic individuals. ${ }^{5}$ Despite the evidence that the incidence of hearing impairment is higher in type 2 diabetes subjects, very little is known about the nature and characteristics of this disability and the specific mechanisms leading to hearing problems in diabetic adults. Studies conducted on the general Caucasian population have shown that cardiovascular disease and its associated risk factors like smoking are associated with hearing loss. ${ }^{6}$ Moreover, some reports on European populations have shown that there is a correlation between audiometric hearing thresholds, worsening glycaemic control and diabetic complications.

In India, a study done by Misra et al (2013) has established that in subjects in early stages of type 2 diabetes with no symptoms of hearing loss there was a mild bilateral, symmetrical, sensorineural hearing loss in $32.65 \%$ of subjects showing that diabetes-induced hypoacusis manifests early. Further follow-up of these patients demonstrated that the hearing loss was irreversible in these patients. ${ }^{7}$ Another report by Baweja et al (2013) done on a sample of type 2 diabetic females showed that a defect in the central auditory pathway is evident in the early stages. A correlation between the duration of diabetes and the extent of hearing loss has been documented by a recent. ${ }^{8}$ study. 
Till now, there are no studies conducted on the Indian population to show the effect of Diabetes on hearing sensitivity comparing the middle-aged and elderly population. Elucidation of the pattern of hearing thresholds across the frequencies and BERA Absolute Latencies and Interpeak Latencies could provide an indication of the mechanism underlying it and thereby be useful in designing clinical rehabilitation strategies and in selection of suitable hearing aids. In the context of this research lacunae and the accumulating evidence on the incidence of hearing loss in type 2 diabetes, the aims of the study were formulated as follows:

1. To obtain the threshold for detection of Hearing Levels in persons with Diabetes across Seven frequencies.

2. To obtain the Absolute Latency and Interpeak Latencies with 21.1 clicks/sec and 63.3 clicks/sec rate at the particular intensity based upon the Pure Tone Threshold.

3. To compare and correlate the Absolute Latencies and Interpeak Latencies between the Diabetics and NonDiabetics of Middle-aged and Elderly Population.

\section{MATERIALS AND METHODS}

\section{Subject Selection}

40 patients (Group A and B) with Type II DM attending the Department of Medicine of Rajiv Gandhi Institute of Medical Sciences, Kadapa, Andhra Pradesh, were included in the study with the co-operation of the Department of Medicine, to elicit Brain stem evoked potentials and analyse them to detect early HL. The study was conducted in the Department of ENT of the same institute during the period of January 2009 and July 2011; 40 subjects (Group C and D) were taken as control group; 40 patients each of these two groups are further subdivided into 20 patients each group depending on their ages as 35 to 58 years and above 58 years.

\section{TEST GROUPS}

Group A: 20 patients of 35-58 yrs. age with DM, Group B: 20 patients aged above 58 yrs. with DM.

\section{CONTROL GROUPS}

Group C: 20 subjects aged 35 to 58 yrs. without DM, Group D: 20 subjects of above 58 yrs. age without DM.

\section{Inclusion Criteria}

Patients diagnosed with Diabetes Mellitus and on treatment (Insulin or oral hypoglycaemic agents) for 5 years or above and/or fulfilment of criteria laid down by the WHO Consultation Group Report, i.e. a fasting venous blood glucose $\geq 126 \mathrm{mg} / \mathrm{dL}$ and/or a $2 \mathrm{hrs}$. post glucose value $\geq 200 \mathrm{mg} / \mathrm{dL}$. Approval of the Institutional Ethics Committee of the RIMS, Kadapa A. P was obtained for the study and written informed consent was obtained from all study subjects.

\section{Exclusion Criteria}

Patients with history of hypertension, patients giving family history of loss of hearing. For control subjects, the inclusion criteria were no history of hypertension and no family history of hearing loss. Subjects with past history of middle ear pathology and other ear pathologies were excluded. Medical examination: Detailed medical history of the patient was obtained and complete ENT check-up was done through otoscopic examination, visual inspection of the pinna and ear canal, tuning fork tests and audiometry so that wax accumulation, ear discharge, foreign bodies, collapsed ear canal condition, peripheral hearing loss could be ruled out. Hearing sensitivity was measured for all 80 subjects with ELKON 3N3 multi-audiometer in a sound treated room. Middle ear pathologies were investigated by performing Immittance Audiometry. There was no middle ear pathology and past history of pathologies in all subjects. Pure Tone Audiometry: This test was conducted according to the ASHA (American Speech-Language-Hearing Association) guidelines. ${ }^{9}$ In this study, the frequencies used in PTA were extended till 12000 Hz. The threshold of hearing is measured by air and bone conduction methods. Pure tones are produced by the electronic audiometer and the intensity of the tones could be elevated or decreased in steps of $5 \mathrm{~dB}$. The quantity of intensity that needs to be raised beyond the normal level provides a measure of the magnitude of hearing impairment at that particular frequency.

The results are charted in graph format called as audiogram. Brainstem evoked response Audiometry: Objective hearing evaluation was done by BERA (Brainstem evoked response Audiometry). BERA is an electrophysiological test procedure, which studies the electrical potential generated at the various levels of the auditory system starting from cochlea to cortex. The filter setting was from $100-3000 \mathrm{~Hz}$. The click rate was set to 21.1 rate, i.e. 21.1 clicks were presented per second which is normal setting for adult population. The total number of clicks presented was 2000. The waves with peaks were elicited and saved with this rate at an intensity level with PTA+50 dB HL. Later the click rate was increased to 63.3 rate, i.e. 63.3 clicks were sent per second which is 3 times more compared to normal settings. The total number of clicks presented were 2000. The waves with peaks were elicited and saved with this rate at an intensity level of PTA $+50 \mathrm{~dB}$ HL. The peak identification was done and located. The latency and interpeak latencies were noted.

\section{Statistical Analysis}

All statistical analyses were performed using SPSS version 15.0 for Windows (SPSS, Inc., Chicago, IL). Data are expressed as mean SD as data were normally distributed. Student ' $T$ ' test (two tailed, independent) was used to compare groups for continuous variables trend, chi square test was used to compare proportions among groups. A p value $<0.05$ was considered statistically significant.

\section{OBSERVATIONS}

Pure Tone Audiometry (PTA) thresholds and BERA intensities in the diabetic and non-diabetic individuals in the middle-aged and elderly group recorded in the Department of ENT. Among the elderly subjects, pure tone averages ranged from $21 \pm 4.47$ to $98.24 \pm 3.03$ in the non-diabetic subjects and $22.5 \pm 3.03$ to $99.71 \pm 1.21$ in the diabetic subjects. Diabetics in general showed a higher degree of hearing loss at all frequencies. However, at $500 \mathrm{~Hz}(\mathrm{p}=0.047), 1 \mathrm{KHz}(\mathrm{p}=0.022), 2 \mathrm{KHz}$ $(\mathrm{p}=0.007), 4 \mathrm{KHz}(\mathrm{p}=0.030)$ and $8 \mathrm{KHz}(\mathrm{p}=0.045)$, the difference in PTA thresholds between diabetic and nondiabetic subjects was statistically significant. Among the middle-aged population, pure tone averages ranged from $14.00 \pm 3.47$ to $41.00 \pm 6.60$ in the non-diabetic subjects and $15.25 \pm 3.79$ to $52.00 \pm 7.84$ in the diabetic subjects. Diabetics in general showed a higher degree of hearing loss at all frequencies. However, at $500 \mathrm{~Hz}(\mathrm{p}=0.013), 1 \mathrm{KHz}(\mathrm{p}<0.001)$, 
$2 \mathrm{KHz}(\mathrm{p}<0.001), 4 \mathrm{KHz}(\mathrm{p}<0.001), 8 \mathrm{KHz}(\mathrm{p}<0.001)$ and 10 $\mathrm{KHz}(\mathrm{p}<0.001)$ the difference in PTA thresholds between diabetic and non-diabetic subjects was statistically significant (Figure I). In both the elderly and the middle-aged group, diabetic subjects showed significantly higher BERA intensities compared to the non-diabetic subjects $(\mathrm{p}<0.008$ in elderly group and $\mathrm{p}<0.001$ in the middle-aged group). Comparison of BERA latencies at 21.1 rates in diabetic and non-diabetic elderly and middle-aged group showed in the elderly group peak I, III and V occurred at a slower rate in the diabetic subjects compared to the non-diabetic subjects. However, Peak I (non-diabetic vs diabetic: $1.31 \pm 0.03$ vs $1.39 \pm 0.03$; $\mathrm{p}<0.001)$ and peak V $(5.84 \pm 0.11$ vs $6.22 \pm 0.13 ; \mathrm{p}<0.001)$ occurred at a significantly slower rate. In the middle-aged group, peak I $(1.14 \pm 0.04$ vs $1.32 \pm 0.04 ; \mathrm{p}<0.001)$, peak III $(3.43 \pm 0.18$ vs $3.90 \pm 0.11 ; p<0.001)$ and peak $V(5.65 \pm 0.10$ vs $6.01 \pm 0.13 ; \mathrm{p}<0.001$ ) occurred at a significantly slower rate in the diabetic subjects compared to the non-diabetic subjects (Figure 1).

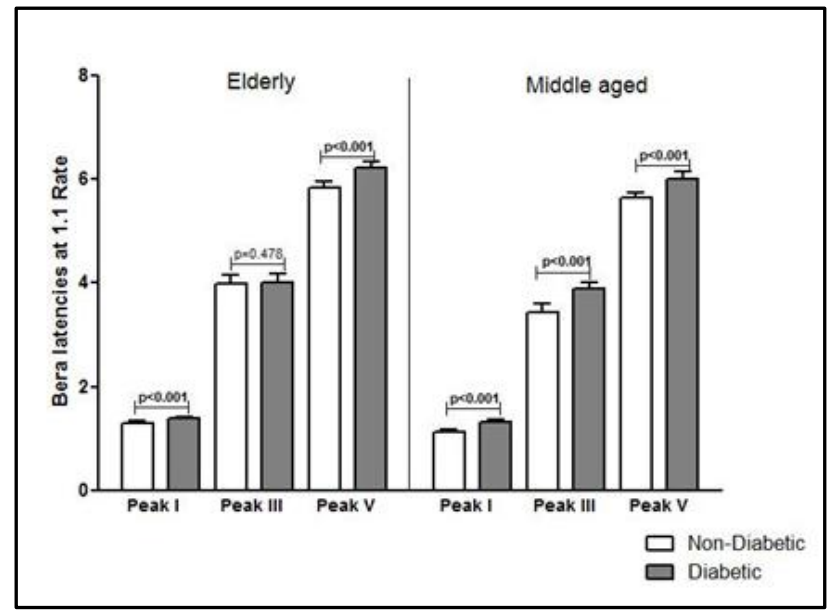

Fig. 1: Comparison of BERA Latencies at 21.1 Rate in Diabetic and Non-Diabetic Elderly and Middle-Aged Group

Comparison of BERA latencies at 63.3 rates in diabetic and non-diabetic elderly and middle-aged group. In the elderly group peak I, III and V occurred at a slower rate in the diabetic subjects compared to the non-diabetic subjects. However, peak I (non-diabetic vs diabetic: $1.63 \pm 0.07$ vs $1.75 \pm 0.05$; $\mathrm{p}<0.001)$, peak III $(5.28 \pm 0.08$ vs $5.80 \pm 0.06$; $\mathrm{p}<0.001)$ and peak V $(6.96 \pm 0.10$ vs $7.16 \pm 0.13 ; p<0.001)$ occurred at a significantly slower rate. In the middle-aged group peak I $(1.33 \pm 0.04$ vs $1.69 \pm 0.02 ; p<0.001)$, peak III $(4.42 \pm 0.08$ vs $5.23 \pm 0.08 ; \mathrm{p}<0.001)$ and peak $\mathrm{V}(5.92 \pm 0.07$ vs $6.65 \pm 0.09$; $\mathrm{p}<0.001$ ) occurred at a significantly slower rate in the diabetic subjects compared to the non-diabetic subjects (Figure 2).

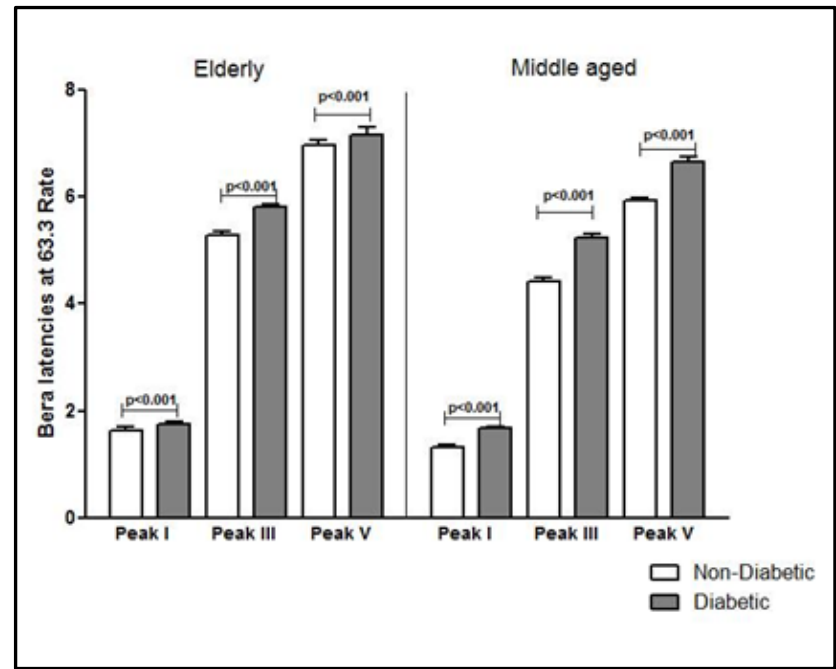

\section{Fig. 2: Comparison of BERA Latencies at 63.3 Rate in Diabetic and Non-Diabetic Elderly and Middle-Aged Group}

Comparison of interpeak latencies at 21.1 rate in diabetic and non-diabetic elderly and middle-aged group. In the elderly group for peak III-V $(1.86 \pm 0.17$ vs $2.19 \pm 0.17$; p <0.001) and peak I-V (4.52 \pm 0.13 vs $4.81 \pm 0.14 ; \mathrm{p}<0.001)$, interpeak latencies were significantly longer compared to the nondiabetic subjects. In the middle-aged group, the interpeak latencies were consistently longer among the diabetics and these differences were significant for the peaks I-III $(2.29 \pm 0.19$ vs $2.59 \pm 0.19 ; \mathrm{p}<0.001)$, the peaks III-V $(2.21 \pm 0.17$ vs $2.11 \pm 0.17 ; \mathrm{p}=0.05)$ and peaks $\mathrm{I}-\mathrm{V}(4.51 \pm 0.11$ vs $4.69 \pm 0.13$; $\mathrm{p}<0.001$ ), (Figure 3).

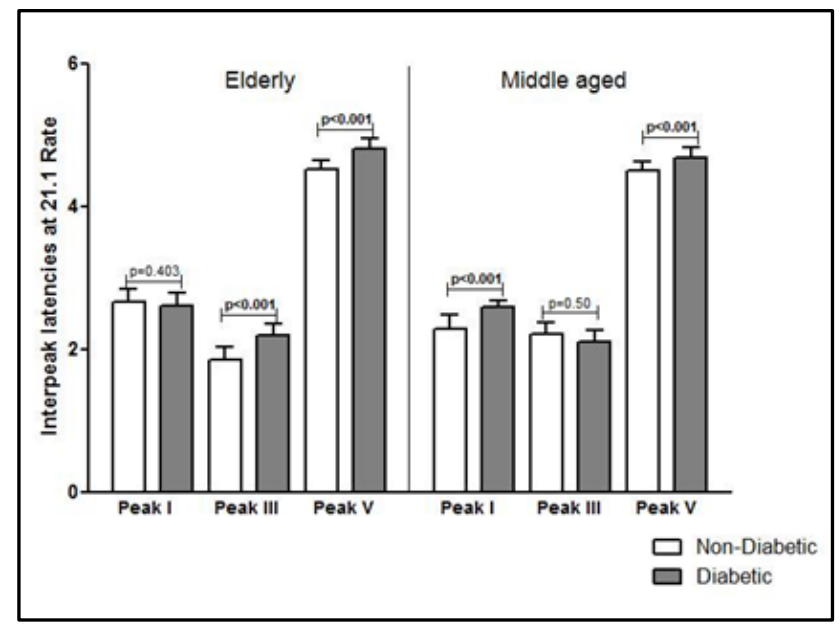

Fig. 3: Comparison of Interpeak Latencies at 21.1 Rate in Diabetic and Non-Diabetic Elderly and Middle-Aged Group 
Comparison of interpeak latencies at 63.3 rate in diabetic and non-diabetic elderly and middle-aged group. In the elderly group for peak I-III ( $3.65 \pm 0.05$ vs $4.05 \pm 0.06$; $p<0.001)$, peak III-V $(1.68 \pm 0.09$ vs $1.36 \pm 0.13 ; p<0.001)$ and peak I-V ( $5.33 \pm 0.08$ vs $5.41 \pm 0.11 ; p=0.013$ ), interpeak latencies were significantly longer compared to the non-diabetic subjects. In the middle-aged group, the interpeak latencies were consistently longer among the diabetics and these differences were significant for the peaks I-III $(3.09 \pm 0.07$ vs $3.54 \pm 0.07$; $\mathrm{p}<0.001)$, the peaks III-V $(1.49 \pm 0.12$ vs $1.42 \pm 0.13$; $\mathrm{p}=0.05)$ and peaks I-V ( $4.59 \pm 0.08$ vs $4.95 \pm 0.12 ; p<0.001)$, (Figure 4$)$.

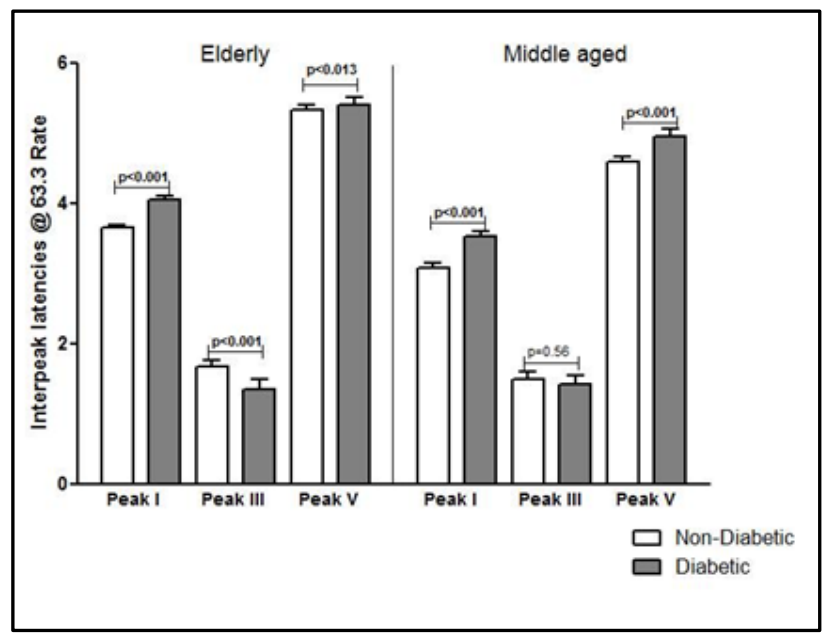

Fig. 4: Comparison of Interpeak Latencies at 63.3 Rate in Diabetic and Non-Diabetic Elderly and Middle-Aged Group

\section{DISCUSSION}

The study makes the following important observations: When the pure tone averages were computed for the diabetics and non-diabetics, it was observed that the diabetic subjects consistently required higher intensities of sound for threshold detection. Particularly, the elderly diabetics needed higher intensities compared to the middle-aged diabetics. In objective hearing evaluation, the threshold for hearing was significantly higher in the diabetic patients when compared to the nondiabetic patients, particularly at higher frequencies. When age-wise comparisons were done, these differences were more pronounced in the middle-aged group compared to the elderly group. The effect of diabetes mellitus on hearing sensitivity and latencies of BERA waves and absolute latencies for slow and fast rates in the middle-aged population and geriatric population were compared.

These parameters were also compared for click rates of $21.1 / \mathrm{sec}$ and $63.3 / \mathrm{sec}$. The results indicated that latencies of all the peaks tended to occur in a delayed manner in the elderly group compared to the middle-aged group. Interpeak latencies in diabetic subjects were longer when compared to nondiabetics showing predominantly significant differences in both elderly and the middle-aged group. However, these Interpeak Latencies were more prolonged in elderly group and this trend was observed both in cases of 21.1 and 63.3 click rates. For the faster click rate (63.3), both the Absolute Latencies and Interpeak Latencies were longer compared to the slower click rates (21.1).

This may indicate that in the older Diabetic group, the transmission in auditory pathway becomes less efficient for higher click rates compared to the Middle-Aged study sample.
Our results are in agreement with the report by Frisnia et al (2006), who observed that the diabetic subjects required higher sound intensities for detection of threshold. ${ }^{10}$ However, our results deviate slightly from the reports of Baweja et $\mathrm{al}^{8}$ conducted on an Indian population. These variations could be attributed to the differences in age and sex distribution of the study samples used in each of these studies. Though the exact mechanisms underlying the auditory pathway defects in diabetes are not elucidated, it is possible that the requirement of high energy and glucose for the complex processing of signals might play a role.

This might also suggest that the cochlea of the ear could be a target organ for the damaging effects of hyperglycaemia. ${ }^{11}$ Increased exposure to glucose even for small periods of time has been shown to initiate a cascade of metabolic reactions that lead to physiologic and anatomical damage to the cochlea. ${ }^{12}$ In the earlier course of type 2 diabetes, though the person may be asymptomatic degenerative changes may begin to appear in the central auditory pathway. ${ }^{13}$ These delicate alterations in the neurodegenerative pathway could be detected in a non-invasive manner with robust accuracy during the early stages of the disease using brainstem auditory evoked potentials. ${ }^{14}$ Considering the variations in lifestyle factors, socioeconomic status and dietary patterns carrying out such studies on a wider scale should be done in future. Furthermore, the pattern of abnormalities in brainstem auditory evoked potentials should be standardized in diabetes mellitus. Considering the escalating incidence rates of diabetes mellitus and the deleterious effects of Diabetes on the hearing sensitivity, it could be concluded that testing the brain evoked auditory potential might be included in the usual list of regular screening procedures like fundus examination, microalbuminuria assessment. 15

\section{CONCLUSIONS}

This is the clinical significance of the study. One of the limitations of the study is the small sample size, which could have biased the study results. But the strength of the study is that this study is the first of its kind comparing middle-aged and elderly diabetic patients without any previous history of hearing impairment. In conclusion the present study shows that in aged diabetic subjects, deleterious ear outcomes could result and this shows that more research is required on the effect of hyperglycaemia on sensory organs. Review of literature shows that here is an association between Hearing losses with Type II DM, but this paper attempts to quantify the losses in such patients, especially belonging to middle and elderly age groups and supports the fact that DM causes SNHL.

\section{ACKNOWLEDGEMENT}

The authors are thankful to all the enrolled subjects without whose active participation this work would not have been possible.

\section{REFERENCES}

1. Saini V. Molecular mechanisms of insulin resistance in type 2 diabetes mellitus. World J Diabetes 2010;1(3):6875.

2. McGavock J, Dart A, Wicklow B. Lifestyle therapy for the treatment of youth with type 2 diabetes. Curr Diab Rep 2015;15(1):568. 
3. Anjana RM, Pradeepa R, Deepa M, et al. ICMR-INDIAB collaborative study group. Prevalence of diabetes and prediabetes (impaired fasting glucose and/or impaired glucose tolerance) in urban and rural India: phase I results of the Indian Council of Medical Research-INdia DIABetes (ICMR-INDIAB) study. Diabetologia 2011;54(12):3022-7.

4. Goldfine AB, Fonseca V. Management of diabetes mellitus in patients with cardiovascular disease in the bypass angioplasty revascularization investigation 2 diabetes (BARI 2D) trial. Circulation 2010;121(22):2447-9.

5. Bainbridge KE, Hoffman HJ, Cowie CC. Diabetes and hearing impairment in the United States: audiometric evidence from the national health and nutrition examination survey, 1999 to 2004. Ann Intern Med 2008;149(1):1-10.

6. Schulz S, Ritter J, Oertel $\mathrm{K}$, et al. Altered autonomic regulation as a cardiovascular risk marker for patients with sudden sensorineural hearing loss. Otol Neurotol 2014;35(10):1720-9.

7. Misra V, Agarwal CG, Bhatia N, et al. Sensorineural deafness in patients of type 2 diabetes mellitus in Uttar Pradesh: a pilot study. Indian J Otolaryngol Head Neck Surg 2013;65(Suppl 3):532-6.
8. Baweja P, Gupta S, Mittal S, et al. Changes in brainstem auditory evoked potentials among north Indian females with type 2 diabetes mellitus. Indian J Endocrinol Metab 2013;17(6):1018-23.

9. Rockville, Asha. American Speech-Language-Hearing Association. Guidelines for Audiologic Screening 1997.

10. Frisina ST, Mapes F, Kim S, et al. Characterization of hearing loss in aged type II diabetics. Hear Res 2006;211(1-2):103-13.

11. Lisowska G, Namyslowski G, Morawski K, et al. Cochlear dysfunction and diabetic microangiopathy. Scand Audiol 2001;52(Suppl):199-203.

12. Sasso FC, Salvatore T, Tranchino G, et al. Cochlear dysfunction in type 2 diabetes: a complication independent of neuropathy and acute hyperglycemia. Metabolism 1999;48(11):1346-50.

13. Baba M, Ozaki I. Electrophysiological changes in diabetic neuropathy: from subclinical alterations to disabling abnormalities. Arch Physiol Biochem 2001;109(3):23440.

14. Uzun N, Uluduz D, Mikla S, et al. Evaluation of asymptomatic central neuropathy in type I diabetes mellitus. Electromyogr Clin Neurophysiol 2006;46(3):131-7.

15. Gupta S, Baweja P, Mittal S, et al. Brainstem auditory evoked potential abnormalities in type 2 diabetes mellitus. N Am J Med Sci 2013;5(1):60-5. 